\title{
Transcriptional transitions in Nicotiana benthamiana leaves upon induction of oil synthesis by WRINKLED1 homologs from diverse species and tissues
}

\author{
Åsa Grimberg ${ }^{1}$, Anders S. Carlsson ${ }^{1}$, Salla Marttila², Rishikesh Bhalerao ${ }^{3}$ and Per Hofvander ${ }^{1 *}$
}

\begin{abstract}
Background: Carbon accumulation and remobilization are essential mechanisms in plants to ensure energy transfer between plant tissues with different functions or metabolic needs and to support new generations. Knowledge about the regulation of carbon allocation into oil (triacylglycerol) in plant storage tissue can be of great economic and environmental importance for developing new high-yielding oil crops. Here, the effect on global gene expression as well as on physiological changes in leaves transiently expressing five homologs of the transcription factor WRINKLED1 (WR/1) originating from diverse species and tissues; Arabidopsis thaliana and potato (Solanum tuberosum) seed embryo, poplar (Populus trichocarpa) stem cambium, oat (Avena sativa) grain endosperm, and nutsedge (Cyperus esculentus) tuber parenchyma, were studied by agroinfiltration in Nicotiana benthamiana.
\end{abstract}

Results: All WR/1 homologs induced oil accumulation when expressed in leaf tissue. Transcriptome sequencing revealed that all homologs induced the same general patterns with a drastic shift in gene expression profiles of leaves from that of a typical source tissue to a source-limited sink-like tissue: Transcripts encoding enzymes for plastid uptake and metabolism of phosphoenolpyruvate, fatty acid and oil biosynthesis were up-regulated, as were also transcripts encoding starch degradation. Transcripts encoding enzymes in photosynthesis and starch synthesis were instead down-regulated. Moreover, transcripts representing fatty acid degradation were up-regulated indicating that fatty acids might be degraded to feed the increased need to channel carbons into fatty acid synthesis creating a futile cycle. RT-qPCR analysis of leaves expressing Arabidopsis WR/1 showed the temporal trends of transcripts selected as 'markers' for key metabolic pathways one to five days after agroinfiltration. Chlorophyll fluorescence measurements of leaves expressing Arabidopsis WR/1 showed a significant decrease in photosynthesis, even though effect on starch content could not be observed.

Conclusions: This data gives for the first time a general view on the transcriptional transitions in leaf tissue upon induction of oil synthesis by WRI1. This yields important information about what effects WRI1 may exert on global gene expression during seed and embryo development. The results suggest why high oil content in leaf tissue cannot be achieved by solely transcriptional activation by WRI1, which can be essential knowledge in the development of new high-yielding oil crops.

\footnotetext{
* Correspondence: Per.Hofvander@slu.se

'Department of Plant Breeding, Swedish University of Agricultural Sciences, Växtskyddsvägen 1, P.O. Box 101, SE-23053 Alnarp, Sweden

Full list of author information is available at the end of the article
} 


\section{Background}

Among different forms of carbon storage, triacylglycerol (TAG; oil) is the most energy dense, yielding more than double amount of energy on a per weight basis as compared to starch. Due to the increased global need for plant oil derived products for food but also for non-food applications to decrease our dependence on fossil oil, the interest in mechanisms for regulation of carbon allocation into oil in plant storage tissues has gained a lot of attention and can be of great economic and environmental importance in the process of developing new high-yielding oil crops $[1,2]$. To be available for TAG synthesis, carbons from sucrose must first be converted to pyruvate through cytosolic or plastidic glycolytic pathways and at some point be imported into the plastid to yield acetyl-coenzyme A (CoA) which feeds fatty acid (FA) synthesis with carbon backbones. FAs are subsequently transported out to the endoplasmic reticulum where TAG assembly takes place [3].

The seed-specific, loss-of-function mutant wrinkled 1 of Arabidopsis thaliana with $80 \%$ reduction in oil content and increased levels of sucrose but not of starch in mature seeds was by enzymatic analyses shown to be impaired in the allocation of carbon into glycolysis [4]. The corresponding gene, AtWRINKLED1 (AtWRI1, At 3g54320), encodes a protein with two APETALA2ethylene responsive element-binding protein (AP2/ EREBP) motifs that is a signature for plant-specific transcription factors involved in a wide range of developmental processes [5, 6]. Gene expression analyses using microarrays have further revealed that some of the down-regulated genes in seeds of the wri1 mutant are involved in glycolysis and FA synthesis, rather than in TAG assembly [7]. Moreover, AtWRI1 has been shown to bind to the AW-box sequence in upstream regions of several genes involved in FA synthesis and glycolysis [8-10].

Homologs to AtWRI1 have been identified in oil-dense embryonic tissues of several other plant species as rape seed (Brassica napus) and maize (Zea mays) [11, 12]. A homolog to AtWRI1 was also associated with the large difference in carbon allocation to oil between oil palm (Elaeis guineensis) and date palm (Phoenix dactylifera) mesocarps with the former mainly storing carbon as oil, the latter as sugar [13]. Today's global plant oil production is mainly dependent on TAG storage capacity in oil palm fruits and the embryos of soybean, sunflower, and oilseed rape [14]. Studies trying to reveal the regulatory mechanisms behind oil accumulation have mainly focused on embryonic tissues of seeds [11, 12, 15], even though fruit mesocarp and seed endosperm tissues have also been studied [13, 16, 17]. However, the potential to produce oil in already high-yielding but not usually oil dense tissues like cereal grain endosperm and underground tuber or root parenchyma, by directing carbon allocation from starch or sugar into oil by genetic engineering, deserves further attention due to the important applied benefits.

Among plant species producing underground tubers, only one is known to have genotypes with high amount of oil (up to $30 \%$ of dry weight), i.e. yellow nutsedge $[18,19]$. Among the cereals, oat is the only species known to store relatively high amount of oil (up to $18 \%$ of grain weight) in the grain endosperm [20,21]. In both these tissues, homologs to the AtWRI1 were expressed during tuber parenchyma and grain endosperm development, respectively (unpublished data, and [22]). Another interesting tissue for potential oil production is from trees due to the vast amount of wood processed in the pulp industry from which FAs are already an important byproduct [23]. Studies on perennial plants have indicated that lipids can accumulate transiently in the cambium upon transition to dormancy [24, 25] and a homolog to the AtWRI1 has been identified in poplar from available genomic resources [26]. Therefore, the role of WRI1-like genes for directing carbon flow into oil synthesis and accumulation could be extended to include diverse types of plant tissues.

In oilseed species like Arabidopsis, oil accumulation is part of the embryo development and seed maturation process which is governed by master regulators such as LEC1, LEC2, FUS3 and ABI3 [15, 27]. WRI1 is linked to this upstream regulatory network and has been shown to be a downstream target and specify the action of LEC2 towards FA synthesis [10, 27]. However, homologs to these upstream regulators were not found in the mesocarp of oil palm, which suggests that the regulatory networks in non-embryo tissues are different to those in developing seed embryos [13]. In this study, we investigated the effect on global gene expression in leaves where oil accumulation was induced by expression of WRI1 homologs originating from diverse species and tissues (Arabidopsis and potato seed embryo, poplar stem cambium, oat grain endosperm, and nutsedge tuber parenchyma) using a transient gene expression system with agroinfiltration of Nicotiana benthamiana. A time study of oil and starch content as well as RT-qPCR and photosynthesis measurements in leaves expressing Arabidopsis WRI1 between one to five days after infiltration was done to further characterize and complement the transcriptional transitions observed at five days after infiltration.

\section{Results and discussion}

\section{All homologs were WRI1-like}

The WRI1 gene from Arabidopsis and four additional homologs from different species representing three dicotyledons and two monocotyledons were used in the present study (for complete cDNA sequences, see Additional file 1). 
These selected homologs are expressed in different types of tissues that all have induction of oil accumulation at certain developmental or temporal stages. The Arabidopsis gene is expressed in the embryo during seed development as is the potato homolog, and the poplar homolog is expressed in the cambium of the tree. The oat homolog is expressed in grain endosperm and the nutsedge homolog in underground stem tuber. From a phylogenetic tree based on homology of fulllength cDNA it was clear that the novel WRI1 homologs of this study were more similar to Arabidopsis WRI1 than to either Arabidopsis WRI2, WRI3, or WRI4 [28] which are more similar to each other than to any of the other presented homologs (Fig. 1). It was also evident from this phylogeny analysis that the two homologs of WRI1 originating from monocot species were more similar to each other than to the dicot WRI1 homologs. The WRI1 orthologs and homologs so far studied have a high degree of identity and similarity on an amino acid level in the region spanning the two AP2 domains but with a high degree of divergence in the $\mathrm{N}$ and C-terminus [29] which was also true for the WRI1 homologs of this study (Additional file 2). However, outside the DNA binding domains there was a high divergence among the different homologs although it was evident that species which are more closely related have a

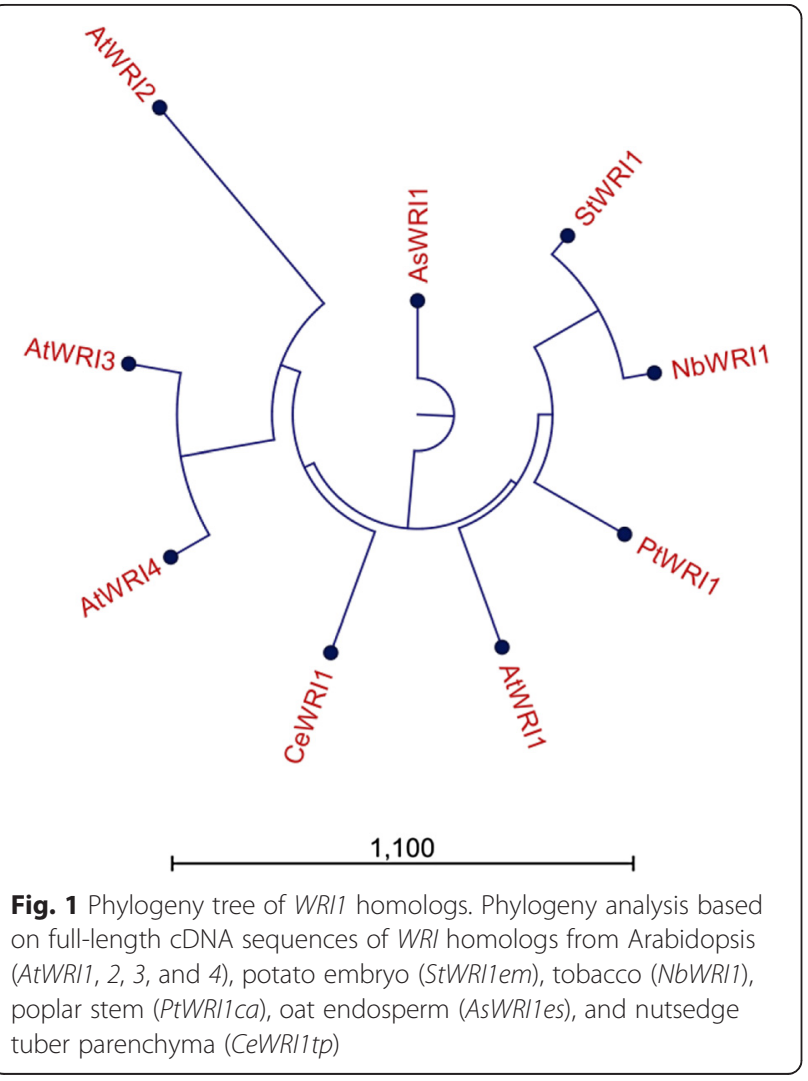

higher degree of amino acid homology and identity. This in particular applies to the derived amino acid sequences of the $N$. benthamiana and $S$. tuberosum homologs of WRI1 with $82 \%$ identity in contrast to the homolog from C. esculentus which had only $45 \%$ identity to that of $N$. benthamiana. The different homologs are hereafter named AtWRI1, StWRI1em, PtWRI1ca, AsWRIles, and CeWRI1tp abbreviated after the species and tissue of origin (embryo, cambium, endosperm, and tuber parenchyma, respectively).

\section{All WRI1-like homologs induced oil accumulation in leaves}

All WRI1 homologs in this study were shown to drastically induce oil (triacylglycerol; TAG) accumulation when transiently expressed in leaves of $N$. benthamiana by agroinfiltration (Fig. 2a, Additional file 3). Leaves expressing the AsWRIles showed the highest oil concentration $(2.2 \%$ of leaf $\mathrm{dw}$ as compared to $0.05 \%$ in transformed control) followed by AtWRI1, StWRI1em, PtWRI1ca, and CeWRI1tp (Fig. 2a). This result shows that the function of WRI1 is highly conserved across species and storage tissues even though not of typical oil-dense embryo type. Also diacylglycerol levels increased in leaves upon expression of the WRI1 homologs (Fig. 2b). Polar lipids (which are important constituents of membranes) were the most abundant in leaves but did not show any difference between leaves expressing the different WRI1 homologs or to transformed control (Fig. 2c). The leaves expressing the WRI1 homologs appeared stressed with chlorosis, in particular for leaves expressing StWRIIem. Dry matter contents of leaves were not significantly different from transformed control (12\%), except for leaves expressing the AsWRI1es with higher dry matter (16\%, see Additional file 4).

The FA composition of TAG changed upon expression of WRI1 homologs with lower proportions of 18:0 and in general higher proportions of 18:1, as compared to transformed control (Fig. 3a). In the polar lipid fraction of leaves, the largest observed differences in FA profile upon expression of WRI1 homologs were in general increased proportions of 18:1 and decreased 18:3 (Fig. 3c).

Since oil accumulation was highest in leaves expressing AsWRIIes and AtWRI1, the localization and structural properties of oil were only analyzed for those (Fig. 4a-c) together with transformed control leaves (Fig. 4d). TEM analysis indicated that the accumulated oil was localized to the cytosol and was most probably present as oil droplets and not as distinct organized oilbodies surrounded by oil-body bound proteins. This is consistent with that no up-regulation of transcripts encoding oil-body proteins was observed in the transcriptome data (see below). However, differences in oil quantity could not be clearly visualized by this method. 


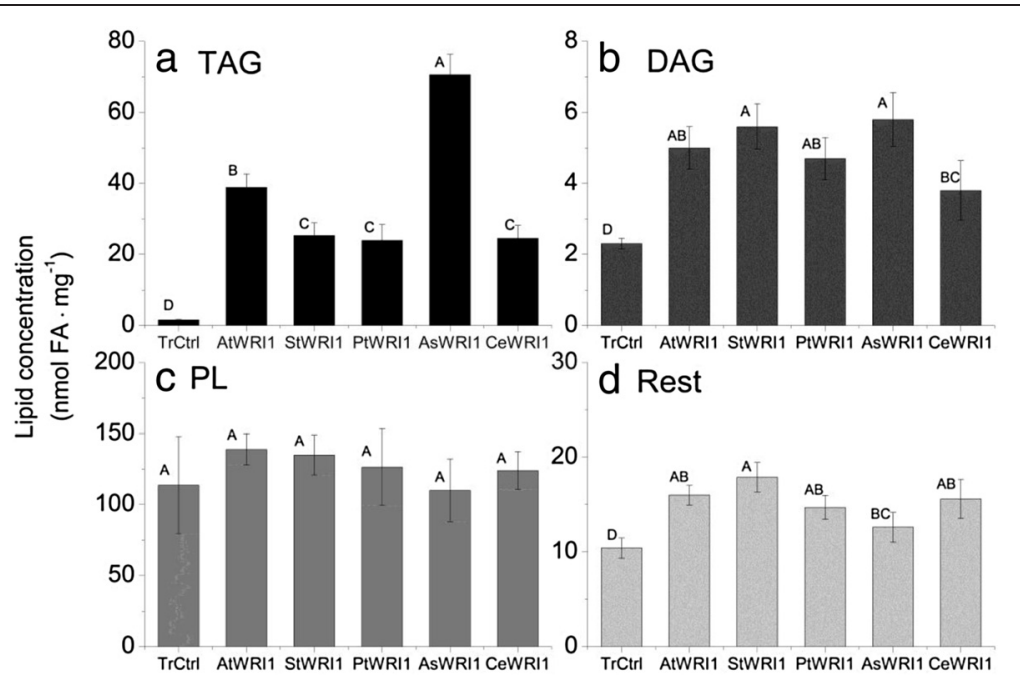

Fig. 2 Leaf lipid concentrations. Concentration of different lipid classes (TAG; triacylglycerol (a), DAG; diacylglycerol (b), PL; polar lipids (c), and other lipids (d)) given as nmol fatty acids (FA) on dry weight basis of N. benthamiana leaves expressing WR/1 from Arabidopsis embryo (AtWR/1), potato embryo (StWR/lem), oat endosperm (AsWR/1es), poplar stem (PtWR/1ca), nutsedge tuber parenchyma (CeWR/1tp), and transformed control (TrCtrl) five days after infiltration. Results are the mean from three biological replicates \pm standard deviation. Letters distinguish significant different means according to Tukey's test at level $P \leq 0.05$

Expression of oleosins in Arabidopsis leaves already expressing phospholipid:diacylglycerol acyltransferase promoted oil accumulation giving $6.4 \%$ oil of leaf $\mathrm{dw}$ and also changed the structural storage form into organized oil-bodies [30], and combining oleosins with WRI1 and diacylglyceroltransferase 1 in $N$. tabacum leaves gave even higher oil content, $15 \%$ by leaf $d w$ [31]. This demonstrates the potential of combining a transcription factor with genes involved in both TAG synthesis and protection against mobilization, for achieving high oil content in leaves. For clarification, the aim of our study was not to achieve the highest oil content possible but instead to map the transcriptional changes induced by WRI1 alone in leaf tissue.
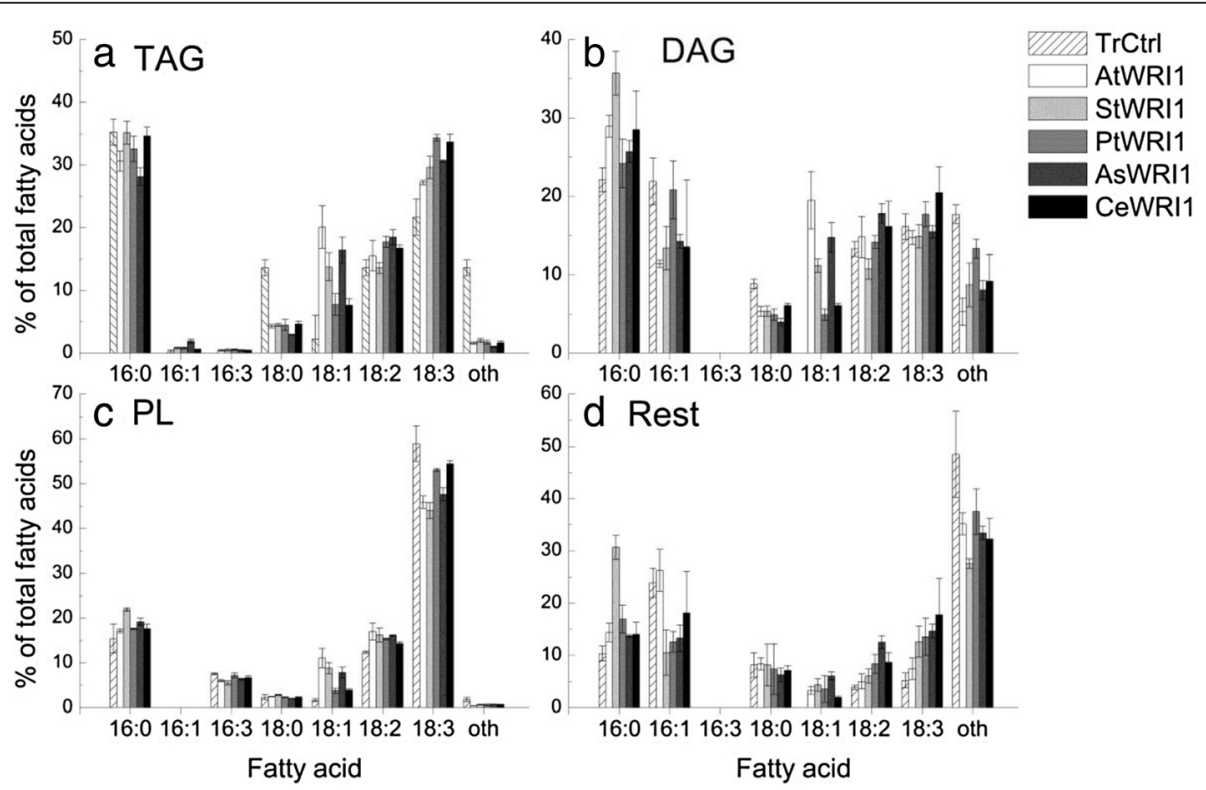

Fig. 3 Fatty acid profiles of lipids. TAG; triacylglycerol (a), DAG; diacylglycerol (b), PL; polar lipids (c), and other lipids (d) in N. benthamiana leaves expressing WR/1 from Arabidopsis embryo (AtWR/1), potato embryo (StWR/1em), oat endosperm (AsWR/1es), poplar stem (PtWR/1ca), nutsedge tuber parenchyma (CeWR/1tp), and transformed control (TrCtrl) five days after infiltration. Results are the mean from three biological replicates \pm standard deviation 

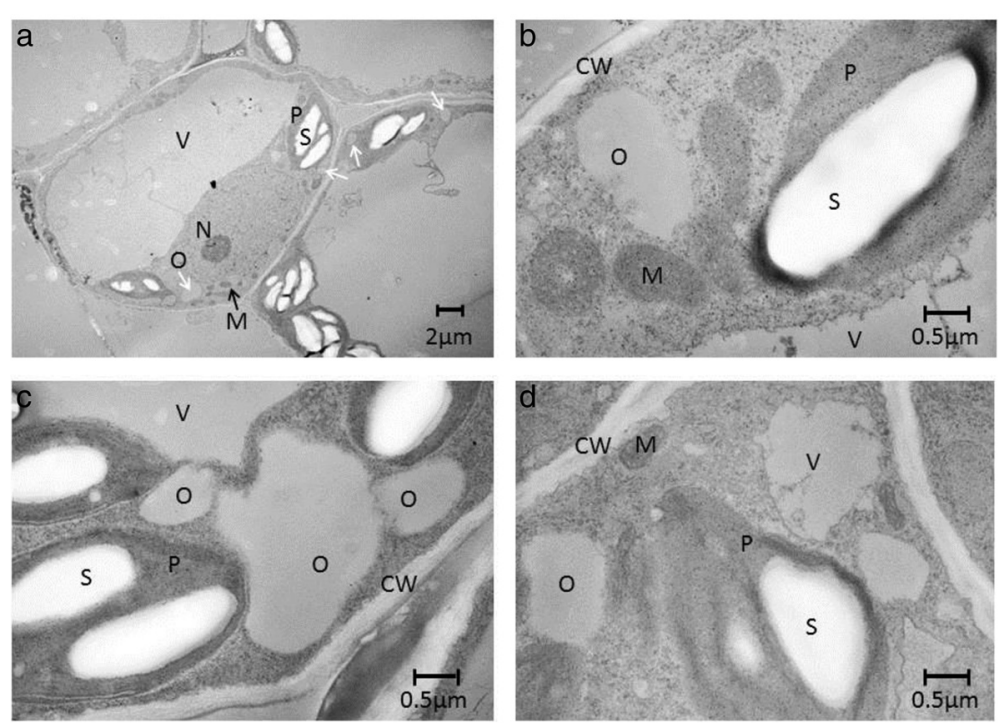

Fig. 4 Transmission electron microscopy. Photos of N. benthamiana leaves expressing WRI1 from oat endosperm (AsWRlles; a, b) and Arabidopsis embryo (AtWRII, c). Transformed control leaf (d) five days after infiltration. CW; cell wall, P; plastid, S; starch, O; oil (white arrows), M; mitochondria, $V_{\text {; vacuole, }}$; nucleus

\section{Transcriptome sequencing data}

Leaves expressing the different WRI1 homologs and transformed control tissue were subjected to transcriptome sequencing five days after agro-infiltration, which is generally used as a suitable time for sufficient transient gene expression and impact on metabolism [32-35]. Upon expression of WRI1 homologs in the N. benthamiana leaf tissue, notable differences in the number of differentially expressed genes as compared to transformed control were observed, but all five homologs induced a similar pattern in core metabolic pathways. Expression of StWRIIem in leaves induced the highest number of differentially expressed genes for both up-regulated and downregulated transcripts, followed by AtWRI1, PtWRI1ca, AsWRI1es, and CeWRI1tp (Additional file 5). However, since the expression levels of the different WRII homologs varied significantly in leaves (RPKM values of CeWRI1tp; 29382, AsWRI1es; 25132, AtWRI1; 7419, StWRI1em; 2712, and PtWRI1ca; 1597), no conclusions regarding speciesspecific differential gene expression induced by those different homologs could be made from this study. Foldchanges of gene expression given here are therefore the averages from leaves expressing the different WRI1 homologs. Interesting to note though, is that the expression levels of the different WRI1 homologs did not correlate either to oil content or number of differential expressed genes in leaves. In fact, the number of differentially expressed genes was instead correlated with phylogenetic similarity of the different WRI1 homologs to the N. benthamiana WRI1, suggesting that the most closely related StWRIIem yielded the highest and
CeWRI1tp the lowest number of differentially expressed genes in leaves.

The lists of differentially expressed genes in leaves (determined according to chosen criteria, see Methods) upon expression of the different WRI1 homologs had in general a similar overall pattern for core metabolic pathways (differentially expressed genes are found in Additional file 6). Here, these general trends are described where the functional equivalence of each gene was assigned according to the closest homolog of Arabidopsis (At-numbers of all genes discussed can be found in Additional file 6). The effect on global gene expression in leaves induced by WRI1 homologs was in general strong with many genes shown to be highly differentially regulated. Some genes were virtually switched 'on', going from almost non-detectable in transformed control tissue to a relatively high expression in WRI1-infiltrated tissue. This illustrates the strong effect of using a constitutively expressed transcription factor to achieve a drastic change of the gene expression program of a certain tissue. The results also reveal notable similarities in the effect on transcriptional regulation exerted by WRI1 homologs coming from such diverse plant species and tissues as were included in this study. It is important to note that it is not possible to distinguish whether the observed effects on gene expression in leaves five days after agroinfiltration were due to direct or indirect effects of WRI1 expression. Furthermore, this study only discusses induced changes at the transcript level and cannot state whether these changes is also correlated to protein levels. 


\section{Carbon directed to the plastid}

The oil-inducing effect of WRI1 in Arabidopsis seeds has been attributed to its enhancing expression of genes encoding enzymes in glycolysis as well as FA synthesis, rather than in TAG assembly $[4,7,10,36]$. Many of the genes, as for biotin carboxylase carrier protein, KASI, enoyl-ACP reductase, ACP, fatty acid desaturase 2, plastid pyruvate kinase, and plastid pyruvate dehydrogenase E1 $\alpha$, which are down-regulated in the Arabidopsis mutant wri1 as compared to in wild-type seeds, are within the set of genes involved in FA synthesis and glycolysis shown to have a bell-shaped expression pattern during wild-type seed development [7]. On the contrary, genes involved in TAG assembly are not differentially expressed in the wri1 seeds and do not belong to the 'bell-shaped group' of genes in the wild-type. Studies on Arabidopsis seeds also showed that the activities of several glycolytic enzymes are much decreased in the wril as compared to wild-type seeds [4, 37]. Therefore, it was not surprising that leaves expressing WRI1 homologs in our study indeed had a much higher expression of genes involved in glycolysis and FA synthesis pathways, as compared to in transformed control (Fig. 5, Additional file 6). WRI1 expression also induced a shift at the transcript level in the organelle distribution of glycolysis with carbon flux being directed to the plastid. Genes encoding enzymes of the upper part of cytosolic glycolysis; sucrose synthase (except for in leaves expressing CeWRI1tp), PPidependent fructose-6-phosphate (Frc-6P) 1phosphotransferase, and Frc-bisphosphate aldolase, and the lower part of plastidic glycolysis; phosphoglycerate mutase, enolase, the $\alpha$ and $\beta$ subunit of pyruvate kinase, and all three subunits (E1-E3) of pyruvate dehydrogenase were highly up-regulated (average fold-changes between 4 and 46, highest for plastidic phosphoglycerate mutase) in leaves expressing WRI1. Up-regulated were also transcripts of the plastid translocator for phosphoenolpyruvate (9fold), thereby probably ensuring cytosolic glycolysis up to phosphoenolpyruvate followed by uptake into the plastid for further plastidic glycolysis. Interestingly, this transcript pattern of organelle shift for glycolysis is very similar to that of Arabidopsis seeds during development [7], indicating that this might be a signature of a plant tissue in transformation from a photosynthetic source to heterotrophic oil accumulation. This pattern is also in agreement with the observed decreased flux of carbon through the lower part of plastidic glycolysis from phosphoenolpyruvate and downwards in developing embryos of the wri1 Arabidopsis mutant as determined by ${ }^{13} \mathrm{C}$ metabolic flux analyses [36].

\section{Increased storage synthesis}

Pyruvate from glycolysis is converted by pyruvate dehydrogenase into acetyl-CoA which is the two-carbon precursor for de novo FA synthesis in the plastid. Many genes encoding enzymes involved in FA synthesis were shown to be up-regulated in leaves expressing the WRI1 homologs (Fig. 5, Additional file 6) again well in agreement with other studies (see above). Transcripts encoding all subunits of acetyl-CoA carboxylase (carboxyl transferase, biotin carboxylase, and biotin carboxylase carrier protein), acyl carrier protein (ACP), malonylCoA:ACP malonyltransferase and 3-ketoacyl-ACP synthase III (KASIII), 3-ketoacyl-ACP synthase I (KAS I) were all up-regulated (4 to 7-fold) in leaves expressing WRI1 homologs. A very high up-regulation was observed for the reductases of 3-ketoacyl-ACP and enoyl-ACP of the fatty acid synthase complex (15-fold). One transcript that was up-regulated 278-fold in leaves expressing the WRI1 homologs, with very low expression in the transformed control tissue, was clearly annotated as a KAS but phylogenetic analyses (based on both DNA and amino acid sequences) did not allow for an obvious designation to KASI or II in Arabidopsis. However, no other KAS genes exist in $N$. benthamiana and therefore this transcript most probably encodes the enzyme activity corresponding to that of KASII in Arabidopsis. The high up-regulation of this transcript indicates the importance of the elongation of 16:0-ACP to 18:0-ACP for increased TAG synthesis.

Genes encoding enzyme activities in TAG assembly were previously not usually associated with WRI1 regulation (see above). However, a few transcripts related to TAG assembly were in this study found to be up-regulated in leaves upon WRI1 expression (Additional file 6). One transcript annotated to represent phosphatidylcholine diacylglycerol cholinephosphotransferase (PDCT) was upregulated (5-fold) in leaves expressing the WRI1 homologs. PDCT is involved in the regulation of TAG composition by catalyzing the inter-conversion of diacylglycerol and phosphatidylcholine [38], and interestingly the encoding gene was previously shown to be down-regulated in the triple mutant of Arabidopsis with reduced expression of WRI1, WRI3, and WRI4 [28]. Transcripts encoding the enzymes phospholipase $\mathrm{C}$ and $\mathrm{D}$ were also up-regulated (average fold changes of 27 and 2, respectively). Both types of lipases are (as also for PDCT) thought to be important for the conversion of phosphatidylcholine to diacylglycerol [3]. Therefore, PDCT and/or phospholipases might therefore be the enzymes defining the observed shift in FA composition of TAG upon induction of oil accumulation. Furthermore, a transcript encoding glycerol-3P dehydrogenase, catalyzing the synthesis of glycerol-3P (the TAG backbone) from dihydroxyacetonephosphate was up-regulated 8-fold. One plastidic isoform of glycerol-3P dehydrogenase in Arabidopsis (At2g40690) was previously shown to be important for the metabolism of plastid-localized glycerolipids [39] and is probably not associated with TAG synthesis (which takes place outside 


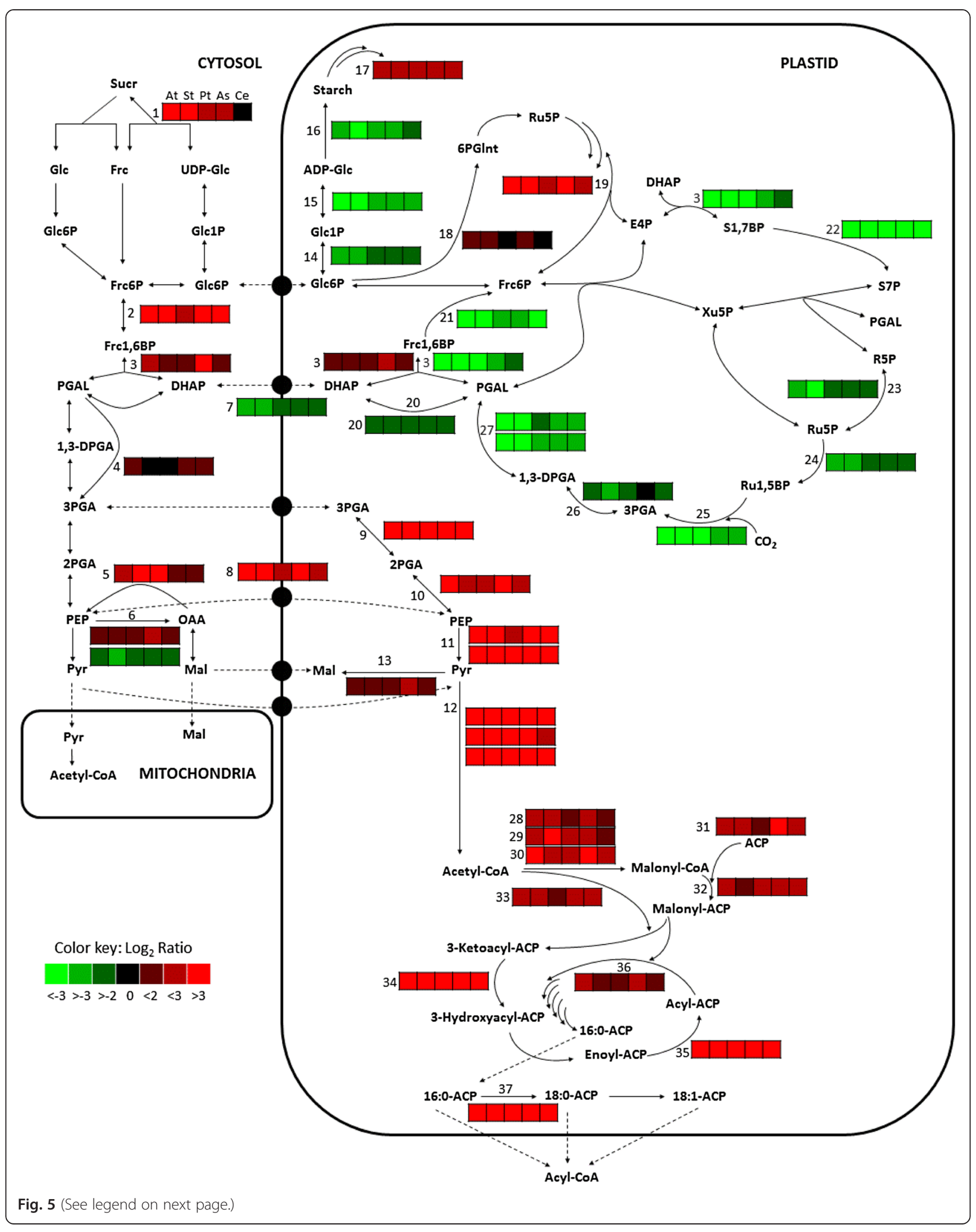


(See figure on previous page.)

Fig. 5 Heat map of differential gene expression involved in central carbon metabolism. Values given are $\log _{2}$ of the ratios between RPKM of leaves expressing WRII and transformed control five days after infiltration. The order of leaf samples expressing the five different WRI homologs are given for the box numbered 1: AtWRI1, StWRIlem, PtWRI1ca, AsWRIles, and CeWR/1tp. Abbreviations: E4P; erythrose 4-phosphate, Sucr; sucrose, Glc; glucose, Frc; fructose, Frc6P; fructose 6-phosphate, Glc6P; glucose 6-phosphate, Frc1,6BP; fructose 1,6-bisphosphate, UDP; uridine diphosphate, Glnt; gluconate, R5P; ribose 5-phosphate, Ru5P; ribulose 5-phosphate, Ru1,5BP; ribulose 1,5-bisphosphate, PGAL; phosphoglyceraldehyde, PGA; phosphoglycerate, DPGA; diphosphoglycerate, PEP; phosphoenolpyruvate, Pyr; pyruvate, OAA, oxaloacetate, Mal; malate, ADP; adenosinediphospho-, S7P; sedoheptulose 7-phosphate, S1,7BP; sedoheptulose 1,7- bisphosphate, Xu5P; xylulose 5-phosphate. Enzymes: 1. Sucrose synthase, 2. PPi-dependent fructose-6-phosphate 1-phosphotransferase, 3. Fructose-bisphosphate aldolase, 4. NADP-dependent glyceraldehyde-3-phosphate dehydrogenase, 5. Phosphoenolpyruvate carboxykinase, 6. Phosphoenolpyruvate carboxylase, 7. Triosephosphate/phosphate translocator, 8. Phosphoenolpyruvate/phosphate translocator, 9. Phosphoglycerate mutase, 10. Enolase, 11. Pyruvate kinase (alpha and beta subunit), 12. Pyruvate dehydrogenase (subunit E1, E2, E3), 13. Malic enzyme, 14. Phosphoglucomutase, 15. ADP-glucose pyrophosphorylase large subunit, 16. Starch synthase 4, 17. $\beta$-amylase, 18. Glucose-6-phosphate dehydrogenase, 19. Transaldolase, 20. Triose-phosphate isomerase, 21. Fructose-1,6-bisphosphatase, 22. Sedoheptulose 1,7-bisphosphatase, 23. Ribose-5phosphate isomerase, 24. Phosphoribulokinase, 25. RubisCO, 26. Phosphoglycerate kinase, 27. Glyceraldehyde-3-phosphate dehydrogenase (subunit A and B), 28. Carboxyl transferase a (CT) subunit of acetyl-CoA carboxylase (ACC), 29. Biotin carboxylase (BC) of ACC, 30. Biotin carboxyl carrier protein 2 (BCCP) of ACC, 31. Acyl carrier protein (ACP), 32. Malonyl-CoA:ACP malonyltransferase, 33. 3-ketoacyl-ACP synthase III (KAS III), 34. 3-ketoacyl-ACP reductase, 35. Enoyl-ACP reductase, 36. 3-ketoacyl-ACP synthase I (KAS I), 37. 3-ketoacyl-ACP synthase II (KAS II)

the plastid). However, the glycerol-3P dehydrogenase shown to be up-regulated in our study (corresponding to At5g40610) was encoding another plastidic isoform [40] which might be important for TAG backbone supply since it is associated with gene expression during seed development in Arabidopis (Additional file 7), and glycerol-3P can pass the plastid envelope through a permease which was recently shown to play an important role for lipid content of Arabidopsis embryos [41]. Interestingly, the gene encoding the plastidic glycerol-3P dehydrogenase found to be up-regulated in our study was also identified to be one of 18 putative target genes of maize (Zea mays) WRI1 [42].

Many genes involved in amino acid metabolism (synthesis, transport and protein processing) were differentially expressed upon expression of WRI1 homologs in our study (Additional file 6) which could possibly indicate increased activities involved with protein storage accumulation. Transcripts encoding vacuolar sorting receptor was 2-fold up-regulated while that of vacuolar processing enzyme as much as 1400-fold up-regulated. Other examples of up-regulated transcripts encoded membrane amino acid transporters (40-fold) and endoplasmic reticulum to Golgi vesicle mediated transport of amino acids (7-fold). Transcripts encoding asparagine synthetase and glutamate synthase was up-regulated (85 and 4-fold, respectively), while on the other hand that of chloroplastic glutamine synthetase was down-regulated (4-fold). Transcripts annotated as amino transferases of aspartate and alanine were up-regulated (2-fold). However, it was difficult to completely dissect the meaning of these differentially expressed genes as pathways were up-regulated or down-regulated during seed development in Arabidopsis [43], which could fit with WRI1 expression although changes in amino acid metabolism and nitrogen remobilization is also consistent with other patterns such as stress or senescence. Changes in expression of genes in amino acid metabolism are also indicative as markers for changes in general metabolism in the leaf tissue [44]. It is interesting to note that increased levels of certain amino acids were actually observed in maize kernels overexpressing the ZmWRI1a [42] which can indicate an association of WRI1 with storage protein synthesis.

\section{Decreased photosynthesis and starch formation}

An important finding in this study was that the expression of WRI1 homologs in leaves induced a severe downregulation of transcripts encoding functions in the photosynthetic apparatus (Additional file 6), both in the light-dependent reactions (subunits and support proteins of photosystem I and II, cytochrome b6f complex, plastocyanin, a leaf-type ferredoxin: $\operatorname{NADP}(\mathrm{H})$ oxidoreductase, subunits of ATP generation complex, 5 to 66-fold changes, see Fig. 6) and the carbon fixation reactions of all three phases of the Calvin-Benson cycle (phosphoribulokinase, Rubisco, and the plastidic forms of phosphoglycerate kinase, glyceraldehyde-3-phosphate dehydrogenase, triose phosphate isomerase, Frc-bisphosphate aldolase, Frc-1,6-bis-phosphatase and ribose-5-phosphate isomerase, 3 to 18 -fold changes, see Fig. 5). Thus the whole Calvin-Benson cycle was down-regulated together with the light harvesting and probably ATP generation of the photosynthetic apparatus (ATP synthase down-regulated 8 -fold). In agreement with this, the transcript encoding the plastid localized triosephosphate/phosphate translocator, with a substrate specificity allowing organelle exchange of dihydroxyacetone-P, glyceraldehyde-3P, and 3-phosphoglycerate [45], was also down-regulated (3-fold) in WRI1 expressing tissue which could indicate a decreased flux of the daily photosynthetically fixed carbon out from the plastid for further sucrose synthesis [46].

One example where functional duality of enzymatic activities became obvious was for Frc-bisphosphate aldolase 


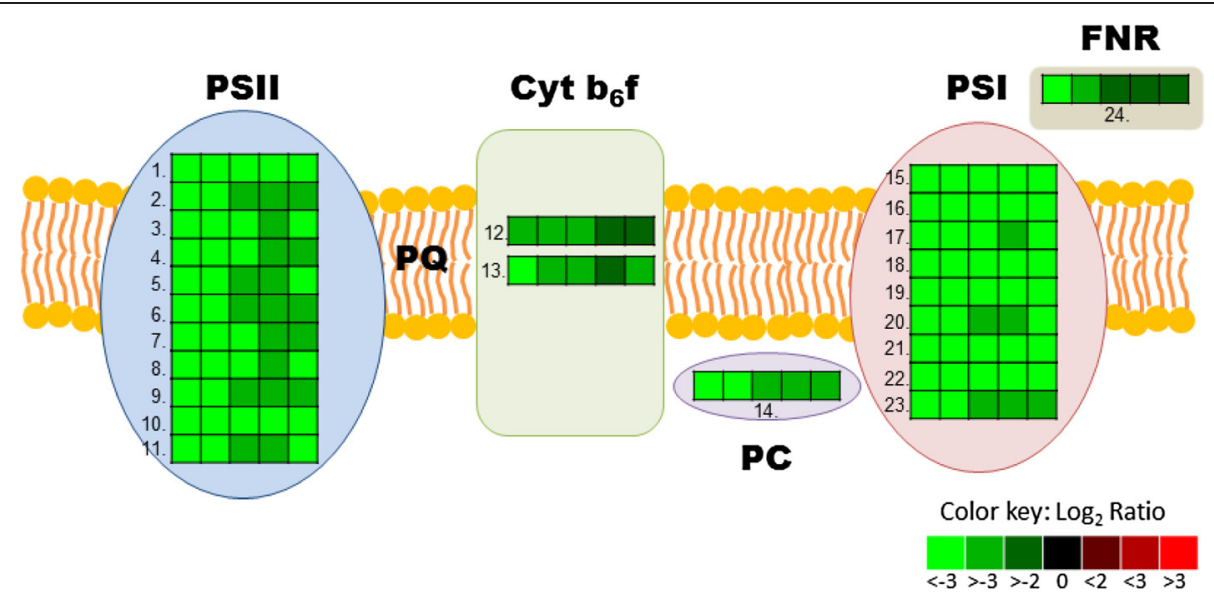

Fig. 6 Heat map of differential gene expression involved in photosynthesis. Values given are $\log _{2}$ of the ratios between RPKM of leaves expressing WRI1 and transformed control five days after infiltration. The order of leaf samples expressing the five different WR/1 homologs are given for the box numbered 1: AtWRI1, StWRI1em, PtWRI1ca, AsWRIIes, and CeWRI1tp. 1. Light harvesting complex photosystem II subunit 6, 2. Photosystem II subunit P, 3. PsbQ subunit of the oxygen evolving complex of photosystem II, 4. Photosystem II subunit PsbX, 5. Photosystem II type I chlorophyll a/b-binding protein, 6 . Photosystem II encoding the light-harvesting chlorophyll a/b binding protein CP26 of the antenna system of the photosynthetic apparatus, 7. Photosystem II $5 \mathrm{kD}$ protein, 8. Involved in the light harvesting complex of photosystem II, 9. Photosystem II BY, 10. Subunit of light-harvesting complex II, 11. Photosystem II type I chlorophyll a/b-binding protein, 12. Rieske FeS center of cytochrome b6f complex, 13. Cytochrome b6f complex subunit, 14. Plastocyanin, 15. Photosystem I subunit E2, 16. Photosystem I subunit F, 17. Subunit $\mathrm{G}$ of photosystem I, 18. Photosystem I, subunit H2, 19. Photosystem I subunit K, 20. Protein predicted by sequence similarity with spinach PsaD to be photosystem I reaction center subunit II, 21. Photosystem I reaction center, sub PSI-N, 22. Photosystem I subunit O, 23. P subunit of Photosystem I, 24. Leaf-type ferredoxin:NADP(H) oxidoreductase

with one up-regulated and one down-regulated transcript (Fig. 5). Since this enzyme catalyzes the reversible conversion of both Frc-bisphosphate (found in both glycolysis and Calvin cycle) and sedoheptulosebisphosphate (only in the Calvin cycle) into dihydroxyacetonephosphate, it can be speculated that the up-and down-regulated transcripts (closest homologs, At2g01140 and At4g38970, respectively) actually represent genes encoding aldolase with importance for glycolysis and the Calvin cycle, respectively.

Transcripts encoding enzymes in starch synthesis (plastidic phosphoglucomutase, the large subunit of ADP-Glc pyrophosphorylase, and starch synthase 4) were downregulated ( 3 to 7 -fold) while that of $\beta$-amylase, important for starch degradation, was instead up-regulated 6-fold (Fig. 5). Increased gene expression and enzyme activity of $\beta$-amylase was previously observed during development of oil-seeds of Arabidopsis and rape indicating the importance of starch degradation to yield carbon precursors for oil accumulation in these species [7, 47]. Starch breakdown and increased glycolysis was also correlated to increased FA synthesis at transcript level in the cambium of aspen upon developmental transition to dormancy [25].

The expression of the different WRI1 homologs in leaves induced down-regulation of many genes involved in chloroplast FA desaturation and very long chain FA (VLCFA) and cutin biosynthesis (Additional file 6). Examples were transcripts encoding FAD5 (responsible for 16:0 desaturation on galactolipids and sulpholipids in chloroplasts), FAD7 (responsible for the desaturation, also in the chloroplast, to 16:3 and 18:3 FAs found on galactolipids, sulpholipids and phosphatidylglycerol), LACS1 (CER8), LACS2 long-chain acyl-CoA synthetases related [48], CER6 (which has a major role in the production of 26 and 28 carbon FAs [49]), and a CER26-like HXXXD acyltransferase (which could have a role in elongation of VLCFA longer than 30 carbons [50]). In addition, genes annotated as coding for modifier functions of cutin were also down-regulated like CER1 which is suggested to be an aldehyde decarbonylase [51], and DCR (defective cuticle ridges), which is a HXXXD acyltransferase [52]. Furthermore, a gene homologous to glycerol-3-phosphate acyltransferase shown to be critical for acyl transfer in cutin biosynthesis [53] was down-regulated. Possibly genes encoding P450 proteins corresponding to functions of importance for cuticle development as CYP86A2 [54] were also among downregulated genes in leaves expressing WRI1 homologs. Among all those, the transcript encoding FAD5 was the most severe down-regulated (53-fold), with other transcripts showing 4 to 7 -fold changes. Interesting and in agreement with our results, a reduction of gene expression encoding one of the chloroplast desaturases, FAD7, was previously observed in leaf tissue expressing AtWRI1 [31]. In the same publication it was noted that in the presence of increased TAG synthesis there was a severe reduction in surface lipids. Our results suggests that the reduction in surface lipids they observed would not be 
due to a competition for substrates but rather a function of reduced gene expression of transcripts related to surface lipid synthesis in response to expression of WRI1.

All these changes induced by WRI1 expression imply that many of the transcripts encoding enzymes giving typical characteristics of a source tissue (photosynthesis, starch synthesis, and leaf surface lipid synthesis) were highly down-regulated, suggesting a shift into a heterotrophic tissue with low photosynthetic contribution. This can probably explain the observed chlorosis of leaves expressing WRI1. It can be speculated that WRI1 mediates repression of photosynthetic source functions also during seed development.

\section{Carbons from oil recycled to plastid}

In oil-accumulating sink tissue such as a seed, the sucrose from photosynthesizing leaves will feed the developing seed allowing for a continuous carbon flow into storage product accumulation. However, when expressing a transcription factor that, directly or indirectly, not only induce oil synthesis but also reduce photosynthesis, the sucrose available for the increased FA synthesis is likely to become limiting. In fact, transcripts encoding an $\alpha / \beta$ hydrolase with TAG lipase activity (yielding glycerol and free FAs) was highly up-regulated (192-fold) while glycerol kinase (thought to play a role for the catabolism of glycerol during post-germinative growth and leaf senescence [55]), and several enzymes involved in $\beta$-oxidation of FAs (acyl-CoA oxidase, multifunctional protein, and 3-ketoacyl-CoA thiolase) were also upregulated 3 to 6 -fold (Additional file 6). Furthermore, a transcript encoding malate synthase, involved in the closely connected glyoxylate cycle that produces malate from degraded FAs, was also highly up-regulated (78-fold). To be available for FA synthesis, malate can then either be translocated into the plastid in exchange for phosphate $[56,57]$ where it can be converted to pyruvate by malic enzyme, or cytosolic conversion of malate by malate dehydrogenase and phosphoenolpyruvate carboxykinase yields phosphoenolpyruvate which can be transported into the plastid by the phosphoenolpyruvate/phosphate translocator. Several transcripts encoding enzyme activities in both these routes (phosphoenolpyruvate carboxykinase, phosphoenolpyruvate/phosphate translocator, and malic enzyme, Fig. 5) were up-regulated (3 to 9-fold) in WRI1 expressing leaf tissue as compared to transformed control. Our results therefore suggest that the new sink-like tissue induced in the leaf by expression of WRI1 is experiencing carbon source limitations. In the absence of sucrose import this probably triggers the up-regulation of transcripts encoding enzymes in both TAG and FA degradation in the leaf in attempt to yield carbons that can be recycled back, either as malate or phosphoenolpyruvate, into glycolysis. An alternative or additional explanation could be that increased amount of TAG in a leaf mesophyll cell, without proper packaging with oil-body proteins, is more prone to mobilization which induces up-regulation of transcripts encoding enzymes of TAG degradation. This will in turn yield free FAs that can be toxic to the cell if accumulating [58], which can explain the up-regulation of transcripts encoding functions in FA degradation. (In connection to this, see discussion below regarding the timestudy of free FA levels in leaves).

As mentioned earlier, the highest total oil content of leaves achieved in our study ( $2.2 \%$ by dw, Fig. 2$)$ was relatively low as compared to in other studies where the stable expression of combination of the transcription factor WRI1 with other genes involved in TAG assembly and storage (the 'push-pull-protect' approach) showed that much higher oil content is possible to achieve (15\% by $\mathrm{dw}$ ) in $N$. tabacum leaves [31]. Interestingly, the promoter chosen for AtWRI1 expression in that study was that of Arabidopsis rubisco, with the rational of achieving a diurnal expression pattern. However, the downregulation of photosynthesis and the Calvin-Benson cycle induced upon WRI1 expression in this study suggests that WRI1 itself might be attenuating the expression of WRI1 from the rubisco promoter. It is possible that this plausible self-regulating effect of the AtWRI1 expression could be another key to the high levels of TAG accumulated without apparent negative effects on plant vigor in that study.

\section{Additional differentially expressed genes of potential importance for seed development}

A number of genes were differentially expressed in leaves upon WRI1 expression where the closest homologs in Arabidopsis are expressed during seed development or corresponding proteins are of importance for proper seed development. For example, transcripts annotated as encoding trehalose-6-phosphate synthase were up-regulated 30-fold (Additional file 6). Trehalose6-phosphate is thought to be a signal of sugar status in plant storage tissues and the synthase is of crucial importance for embryo development in Arabidopsis [59-61]. Transcripts encoding proteins of completely unknown function but where the closest Arabidopsis homolog is expressed during seed development were found, exemplified by a transcript annotated as closest homolog to At2g22660 (DUF1399) (Additional file 8) which was highly up-regulated (16-fold) in WRI1 expressing tissue (Additional file 6).

For another transcript which was also highly upregulated (215-fold), the closest homolog in Arabidopsis was Atlg43850 encoding SEUSS, a transcriptional adaptor of importance for embryonic development [62]. There were additional transcripts annotated as nucleic acid binding or transcription factors although their functions 
are unknown and gene families so large that closest homologs in Arabidopsis were difficult to discern. Only one transcript related to genes encoding oil body proteins was up-regulated by the WRI1 homologs which closest homolog was Atlg178101 encoding a putative aquaporin [63].

\section{Time study of leaves expressing AtWRI1}

Some of the transcriptional transitions observed in leaves induced, directly or indirectly, by the transient expression of all the different WRI1 homologs were unexpected, such as the effect on transcripts encoding photosynthesis, starch metabolism, and fatty acid degradation. Therefore, the effects of the transient expression of AtWRI1 in N. benthamiana leaves were physiologically characterized in more detail in a time study from one to five days after infiltration (DAI). Non-transformed control leaves were also analyzed in this time-study to confirm it was not physiologically different to transformed control (with $p 19$ and GFP constructs only). The expression levels of AtWRI1 and a subset of genes encoding enzymes selected as 'markers' for core metabolic pathways (glycolysis, Calvin cycle, starch synthesis, and fatty acid degradation) were also determined using RT-qPCR (Fig. 7). Gene expression levels were determined relative to the gene L23 that was recently suggested to be a suitable reference in $N$. benthamiana leaves [64] and which also from our transcriptome data set was confirmed to be stable between treatments and with an expression level in a suitable range in comparison to target genes.

\section{Increased AtWRI1 expression and TAG content from two DAI}

AtWRI1 expression was barely detectable in leaves at one DAI but was much increased already from two DAI as compared to in transformed control and nontransformed control, and was only slightly further increased (but not statistically significant) at five DAI (Fig. 7a). Triacylglycerol content of leaves expressing AtWRI1 was significantly increased already by two DAI, even though it continued to increase up to five DAI (Fig. 8). This gives insight to how fast the metabolic effect generated by a constitutively expressed transcription factor in leaves can actually be observed. The expression level of the gene encoding pyruvate dehydrogenase (used as a marker for glycolysis) showed a pattern very similar to that of AtWRI1 (Fig. 7b) which is well in accordance with that this gene is considered to be a direct transcriptional target of AtWRI1 [10].

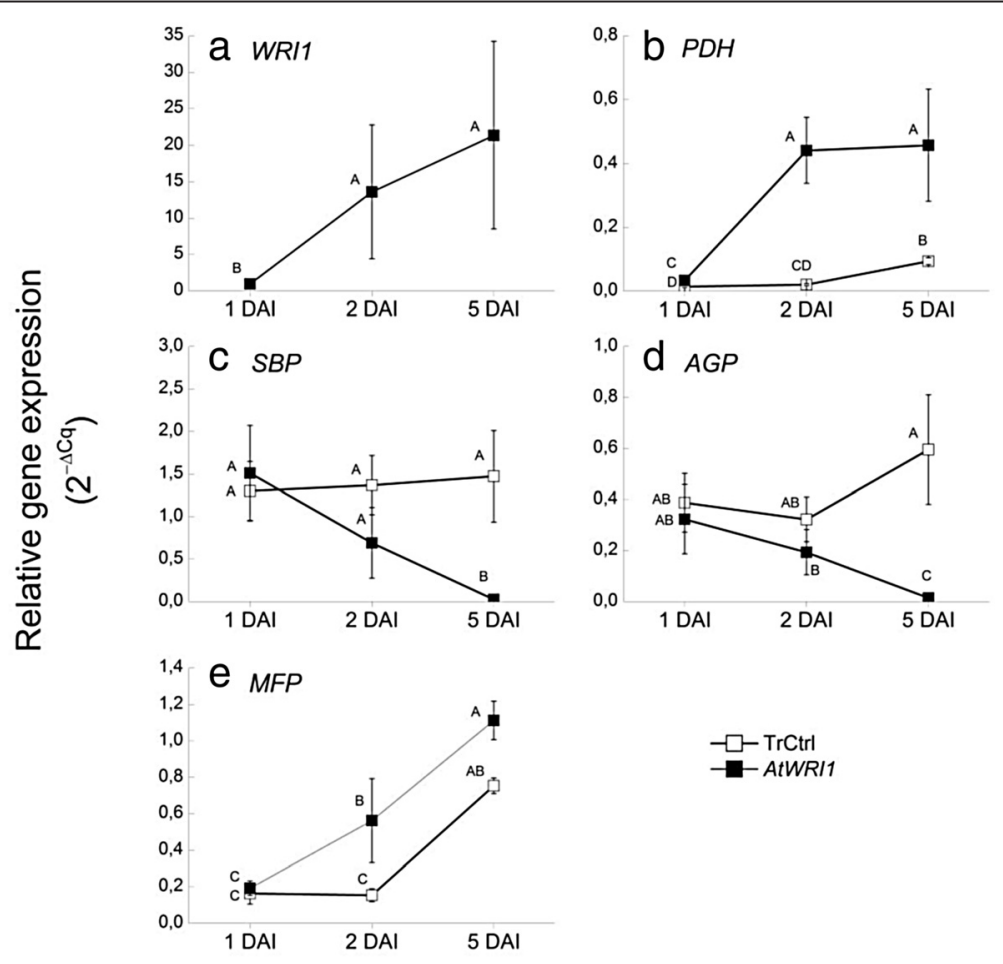

Fig. 7 RT-qPCR time study of leaves expressing AtWR/1. Expression levels of AtWR/1 (a) and a subset of genes encoding enzymes selected as 'markers' for core metabolic pathways: PDH; pyruvate dehydrogenase for glycolysis (b), SBP; sedoheptulose-bisphosphatase for Calvin cycle (c), AGP; ADP-Glc pyrophosphorylase for starch synthesis (d), and MFP; multifunctional protein for fatty acid degradation (e) from one to five days after infiltration (DAI). Gene expression levels $\left(2^{-\Delta C a}\right)$ were determined relative to the reference gene L23. TrCtrl; transformed control. Results are the mean from three biological replicates \pm standard deviation. Letters distinguish significant different means according to Tukey's test at level $P \leq 0.05$ 


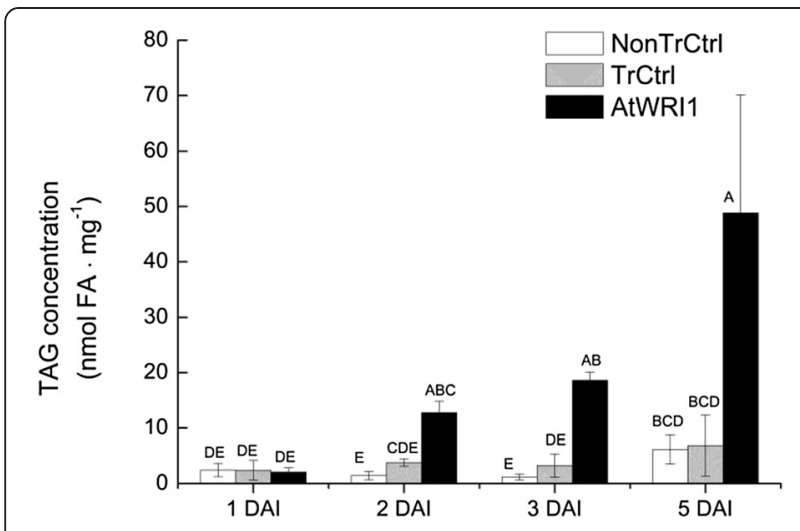

Fig. 8 Time study of triacylglycerol concentrations in leaves expressing AtWR/1. Given as nmol fatty acids (FA) per mg dry weight (dw) from one to five days after infiltration (DAI). TrCtrl; transformed control, NonTrCtrl; non-transformed control. Results are the mean from three biological replicates \pm standard deviation. Letters distinguish significant different means according to Tukey's test at level $P \leq 0.05$

\section{Decreased photosynthetic capacity of leaves}

Fluorescence efficiency measurements clearly showed decreased photosynthetic capacity in leaves expressing AtWRI1 as compared to both non-transformed leaves and transformed control from three DAI (Fig. 9) which was well in agreement with the observed severe downregulation of many transcripts encoding different parts of the light-dependent reactions of photosynthesis (see above) as well as down-regulation of the gene used as a marker for the Calvin cycle (sedoheptulose-bisphosphatase, Fig. 7c). This result also verified that transformed control (leaves infiltrated with only $p 19$ and GFP constructs, see methods) was physiologically indistinguishable from non-transformed control (Fig. 9). Starch content was measured over time (Additional file 9) but no significant decrease could be detected. This implies

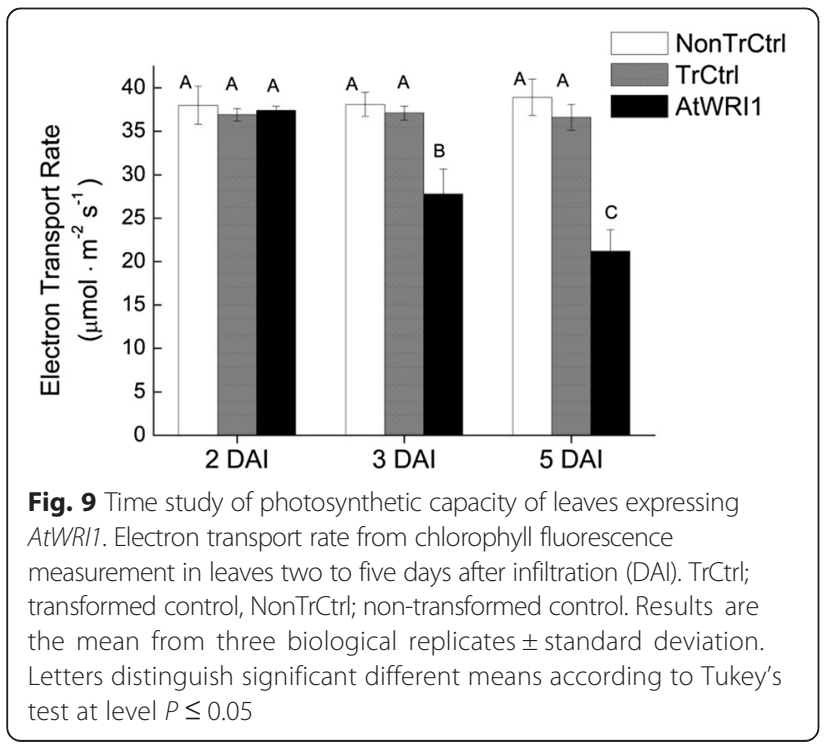

that the previously described transcriptional changes observed at five DAI that were involved in photosynthesis reactions (light harvest reactions and Calvin cycle) and starch metabolism (decreased synthesis and increased degradation) were not detectable at the metabolic level of starch, at least not over the time period studied. In agreement with this, the level of the transcript encoding ADP-Glc pyrophosphorylase (catalyzing the first committed step in starch synthesis) was only statistically distinguishable from transformed control as late as by five DAI (Fig. 7d). The effect on starch levels in leaves expressing WRI1 could therefore probably not be expected until after the time period studied (i.e. after five DAI).

\section{No increase in levels of free FAs in leaves}

The observed up-regulation of transcripts encoding enzymes involved in TAG and FA degradation could, as discussed above, have several different explanations. It was of interest to know if leaves expressing WRI1 had increased levels of free FAs which can have negative physiological effects [58]. This could be a reason to the observed up-regulation of transcripts encoding functions in FA degradation. From total lipid analysis in our study it was recognized that all lipids except the polar fraction were increased upon expression of WRI1 homologs in leaves five DAI (Fig. 2). The increase of lipids in the 'Rest' fraction could therefore potentially include increases of free FAs, even though not visually detectable on TLC plates (Additional file 3). However, analysis of free FAs in leaves expressing AtWRI1 showed no significant increase between one and five DAI (Additional file 10), implying that free FA levels were either not increased, or degraded in pace with the release of free FAs thereby keeping levels low. The gene expression level of multifunctional protein, used as a marker for $\beta$-oxidation of FAs, was significantly increased in leaves expressing AtWRI1 already from two DAI and onwards, as compared to transformed control (Fig. 7e) which suggests a relatively fast response to the WRI1 mediated changes in the leaf.

\section{Conclusions}

This study gives for the first time an overview on the transcriptional transitions in leaf tissue upon expression of the transcription factor WRI1. Expression of WRI1 homologs from five diverse species and tissues in leaf all induced oil accumulation and the same general shifts in gene expression programs with down-regulation of transcripts encoding typical source tissue functions and upregulation of those encoding typical sink tissue functions. The severe down-regulation observed for transcripts encoding photosynthetic functions was confirmed physiologically with a significant decreased photosynthetic 
capacity observed of leaves expressing AtWRI1. Altogether this study revealed a wider range of effects caused, directly or indirectly, by WRI1 than previously suggested. These changes are reminiscent of the transcriptional and metabolic transitions detected between the vegetative phases of seed development and maturation in Arabidopsis; from high photosynthetic contribution, starch accumulation, and cytosolic metabolism of phosphoenolpyruvate, to oil biosynthesis with decreased photosynthesis and plastid uptake and metabolism of phosphoenolpyruvate, during the course of seed development $[7,15,44,47]$. However, leaves with WRI1 expression did not only accumulate oil but transcriptional changes indicated that functions encoding TAG and FA degradation was up-regulated, probably in attempt to feed the increased need for carbons into FA and oil synthesis thus creating a futile cycle. This could explain why high oil content in leaf tissue cannot be achieved by high and constitutive transcriptional activation by WRI1. Most likely this response is due to an incomplete shift from source to sink tissue and a lack of induction of sucrose import.

It is intriguing that expression of WRI1 alone can induce these large transcriptional and physiological changes in a leaf, and future studies need to functionally dissect the different roles of WRI1 and upstream transcriptional actors previously suggested to take part in seed development in general [65]. Moreover, identification of the regulatory networks present in other types of oil-storing plant tissues in which WRI1 is known to act in will be of utmost importance for the development of high-yielding oil crops in the future.

\section{Methods}

\section{Plant material}

All plants were grown in controlled growth chambers (Biotron, SLU-Alnarp, Sweden) under fluorescent light $\left(200 \mu \mathrm{mol} \cdot \mathrm{m}^{-2} \cdot \mathrm{s}^{-1}\right)$ and with the following conditions: $N$. benthamiana; $15.5 / 8.5 \mathrm{~h}$ light/dark photoperiod with $25 / 20{ }^{\circ} \mathrm{C}$ at $60 \%$ humidity. Oat (Avena sativa L., cv. Matilda, Lantmännen, Svalöv, Sweden) with $10 \%$ oil in grains; $16 / 8 \mathrm{~h} \mathrm{light/dark,} \mathrm{at} 21 / 18{ }^{\circ} \mathrm{C}$ at $60 \%$ humidity. Yellow nutsedge (Cyperus esculentus L. var. sativus Boeck) was grown in an aeroponic system as previously described [18].

\section{Cloning of genes}

WRI1 homologs from oat and nutsedge were cloned from grain endosperm and tuber parenchyma, respectively. Oat endosperm was squeezed out from grains (from which the embryo and scutellum had first been removed to minimize tissue contamination) at mid-stage of development during active endosperm oil deposition (approximately 14 days post anthesis). Nutsedge tubers were harvested 15 days after the onset of tuber initiation during active oil deposition [18]. Total RNA was extracted from tissues using Plant RNA Reagent (Invitrogen, Carlsbad, USA) from which cDNA was synthesized and amplified using available kits either with a 5'-3'RACE approach (for oat, Clontech, Mountain View, USA) or a standard procedure using modified primers to later be used for 454 sequencing (for nutsedge, Invitrogen, Carlsbad, USA). Gene specific primers were designed based on contig transcript sequences annotated to represent homologs to AtWRI1 from previous 454sequencing [unpublished results, 22]. The AtWRI1 from embryo as well as the two homologs from potato and poplar were synthetically ordered (Eurofins, Ebersberg, Germany) based on available genomic resources. Fulllength WRI1 homologs were individually inserted to binary vectors (pART27, pK2GW7, or pXZP393) behind the $35 \mathrm{~S}$ promotor and transformed into Agrobacterium tumefaciens (strain GV3101). The gene constructs with $p 19$ viral suppressor protein to improve gene expression and GFP for positive control and visual localization of gene expression were kindly provided by $\mathrm{Dr} \mathrm{C}$. Wood [35]. Full length cDNA sequences of the respective WRINKLED homologs were subjected to CLUSTAL 2.1 Multiple Sequence Alignment with default settings and circular cladogram created by Tree construction using Neighbor Joining and Bootstrapping with 1000 replicates on CLC Main Workbench 7.1.6 (CLC bio, Aarhus, Denmark).

\section{Agroinfiltration of tobacco leaves}

Agroinfiltration of leaves (six weeks old, $6 \mathrm{~h}$ into the light period) was done as previously described by Wood et al. [35]. The WRI1 homologs were individually combined with $p 19$ and GFP cultures, the transformed control only contained $p 19$ and GFP. Only one leaf per plant was used and identified to represent the same developmental stage (approximately $7 \mathrm{~cm}$ in diameter), and three biological replicates were used per construct combination. All chemicals were from Duchefa (Haarlem, The Netherlands) or Sigma-Aldrich (St Louis, USA). Culture mixtures of approximately $3 \mathrm{~mL}$ were infiltrated into the leaf, and plants were then incubated for five days in growth chambers. Only the leaf area visually identified to show GFP expression under UV-light was used for sampling ( $6 \mathrm{~h}$ into the light period) and was split into two; one for RNA extraction $(\sim 150 \mathrm{mg}$ FW) and the other for lipid analyses ( $900 \mathrm{mg}$ FW). For the time-study of leaves expressing AtWRI1, the leaf area with GFP expression was instead divided into three; one for starch analysis, one for RNA extraction, and one for lipid analysis. Samples were snap frozen in liquid nitrogen and stored in $-80{ }^{\circ} \mathrm{C}$ until further analyses. Prior to sampling, photosynthetic capacity of 
leaves was measured as electron transport rate using a Mini-PAM chlorophyll fluorometer (Walz, Effeltrich, Germany).

\section{Lipid analyses}

Leaf samples were freeze dried and dry weight determined. Total lipids were extracted [66] and aliquots corresponding to $10 \mathrm{mg} \mathrm{dw}$ were separated using thin layer chromatography and quantified using gas chromatography with methyl-heptadecanoate as internal standard as previously described [67].

\section{Starch analyses}

Dried plant material $\left(80{ }^{\circ} \mathrm{C}\right.$ for $\left.48 \mathrm{~h}\right)$ was ground with mortle and pestle to a fine powder. Starch concentration was determined enzymatically on approximately $20 \mathrm{mg}$ leaf $\mathrm{dw}$ per biological replicate using the Total starch determination kit (K-TSTA 07/11, Megazyme, Wicklow, Ireland) according to recommended procedure except that samples were first washed with $80 \%$ ethanol at $80{ }^{\circ} \mathrm{C}$ to remove soluble sugars.

\section{Transmission electron microscopy}

Leaves of $N$. benthamiana expressing the oat and Arabidopsis WRI1 homologs where chosen for transmission electron microscopy (TEM). Leaf samples were taken five days after infiltration, $6 \mathrm{~h}$ into the light period. One leaf sample was used for both TEM and lipid analyses to determine TAG content as described above (approximately $1 \%$ of DW for both AtWRII and AsWRI1 leaf samples). Small pieces were cut from the leaf under fixation medium ( $2 \%$ paraformaldehyde $(w / v), 2.5 \%$ glutaraldehyde $(\mathrm{v} / \mathrm{v})$ in $0.1 \mathrm{M}$ Sorensen's sodiumphosphate buffer $\mathrm{pH}$ 7.2) to minimize air contact. Fixative was infiltrated into leaf pieces by repeated vacuum pumping in a desiccator. Further processing for TEM was performed as described previously [68].

\section{RNA extraction and sequencing}

Total RNA from frozen leaf material was extracted by homogenizing material in Plant RNA Reagent according to instructions (Invitrogen, Carlsbad, USA) using a plastic pestle. RNA integrity and concentration was measured using Experion RNA StdSens analysis kit (BioRad, Hercules, USA) before pooling proportional RNA amounts from two biological replicates. Total RNA was DNAse treated (Turbo DNase, Ambion, Carlsbad, USA), diluted to a final concentration of $500-1000 \mathrm{ng} \cdot \mathrm{\mu L}^{-1}$, and sent for Illumina sequencing (BGI, Shenzhen, China). Preparation of libraries was performed at BGI according to their routines and sequencing was done on the Illumina HiSeq 2000.

\section{Bioinformatics and data analyses}

Quality passed reads from the respective libraries were mapped to downloaded Niben genome v0.4.4 transcripts (ftp://ftp.solgenomics.net/genomes/Nicotiana_benthamia na/annotation/Niben044). Total number of reads ranged from 22,801,552 to 24,386,511 for leaves expressing PtWRI1ca and CeWRI1tp, respectively, with leaves expressing the other WRI1 homologs having number of reads in between those. Proportions of mapped sequenced reads to the $N$. benthamiana reference genome ranged from at lowest $63.8 \%$ to highest $79.6 \%$ for leaves with AsWRIles and StWRIlem, respectively. Pairwise comparison (ratio) of RPKM (reads per kilobases and million mapped reads) values of each transcript in WRI1 homolog expressing leaf tissue to that in transformed control yielded an initial file containing transcripts where FDR $\leq$ 0.001 and $\log _{2}$ ratio $\geq 1$ ). Differential gene expression is given as $\log _{2}$ of ratios of RPKM in leaves expressing the WRI1 homologs as compared to transformed control $\left(\log _{2}\right.$ ratio of +3 means that a transcript is $2^{3}=8$ times higher expressed). Transcripts showing a differential expression as compared to transformed control with a $P$ value close to zero $\left(<10^{-307}\right)$ were extracted to an individual table (Additional file 6 with $\log _{2}$ ratios in bold font) in which also differential expression values for all other leaf samples were included (even though having a $P$-value above this threshold, $\log _{2}$ ratios in italic font in Additional file 6). For all genes discussed in this study, the annotation made by BGI was complemented with the closest homologs of $N$. benthamiana transcipts to Arabidopsis genes [69].

For determination of WRI1 expression, respective WRI1 sequences used for infiltration were introduced among Niben genome v0.4.4 transcripts in a new reference file. Illumina sequence reads representing transcripts for each of the leaf samples with expressed WRII homologs were subsequently mapped to the new reference file using CLC Genomics Workbench 7.0.4 (CLC bio, Aarhus, Denmark) using the RNA-Seq analysis module at default settings.

\section{RT-qPCR}

Frozen leaf samples taken from the time-study on leaves expressing AtWRI1 (three biological replicates) were ground to a fine powder in $1.5 \mathrm{~mL}$ plastic tubes using metal beads in a mixer mill (MM400, Retsch, Haan, Germany) with care taken to not let material thaw at any time. Total RNA was extracted and $10 \mu \mathrm{g}$ was DNase treated (using the same kits as described above). For cDNA synthesis, $1.2 \mu \mathrm{g}$ DNase treated total RNA was reverse-transcribed using Superscript III FirstStrand Synthesis Supermix for RT-qPCR (Invitrogen, Life Technologies, Carlsbad, CA, USA). Transcript quantification was made on a BIO-RAD C1000 Thermal Cycler, CFX 96 Real-Time System (California, 
USA) using Maxima SYBR Green/ROX qPCR Master Mix (2X) (Thermoscientific, Life Technologies, Carlsbad, CA, USA), $2.5 \mu \mathrm{L}$ of $1: 10$ diluted cDNA and $0.3 \mu \mathrm{M}$ primers in a final reaction volume of $25 \mu \mathrm{L}$ run in white PCR tubes (Starlabs, SaveenWerner, Limhamn, Sweden) using a program with initial $95^{\circ} \mathrm{C}$ for $10 \mathrm{~min}$ followed by 40 cycles of $95{ }^{\circ} \mathrm{C}$ for $15 \mathrm{~s}, 60^{\circ} \mathrm{C}$ for $30 \mathrm{~s}, 72{ }^{\circ} \mathrm{C}$ for $30 \mathrm{~s}$. Primers used are listed in Additional file 11 where forward primers were designed to span the exon borders (for all except $L 23$ and AtWRI1 for which it was not technically possible or meaningful, respectively) to minimize amplification of genomic DNA. All used primers gave single PCR product as determined by visual inspection of agarose gel electrophoresis. $\mathrm{C}_{\mathrm{q}}$ values were calculated using automatic single threshold in the Bio-Rad CFX Manager and values between 18-30 cycles were regarded an appropriate quantification window. Melting curves for PCR products were inspected to confirm identical melting temperature for each specific product. Expression levels of transcripts were calculated relative to L23 [64] according to the equation Relative Expression $=2^{-\Delta \mathrm{Cq}}$ where $\Delta \mathrm{C}_{\mathrm{q}}=\mathrm{C}_{\mathrm{q}}$ (target) $-\mathrm{C}_{\mathrm{q}}$ (reference). Mean values of three technical replicates where used for each biological replicate.

\section{Statistical analyses of data}

Treatment effects on data obtained from chemical analyses and RT-qPCR were analyzed by analysis of variance (ANOVA) using the general linear model (MINITAB 16; Minitab, State College, PA, USA) in which all factors were fixed. Significant differences between treatments were calculated using pairwise comparisons with Tukey's test at level $P \leq 0.05$. When necessary, data was first transformed by the logarithmic transformation before the ANOVA and Tukey's test to stabilize the variance and obtain an approximately normal distribution of the residuals required for valid statistical inference.

\section{Availability of supporting data}

The sequences used in this study have been submitted to the Sequence Read Archive at NCBI (Accession numbers: SRX1079394 (transformed control leaves), SRX1079397 (leaves expressing AtWRI1), SRX1079426 (StWRI1em), SRX1079428 (PtWRI1ca), SRX1079429 (AsWRI1es), and SRX1079431 (CeWRI1tp).

\section{Additional files}

Additional file 1: Complete CDNA sequences of WRI1 homologs. Sequences are from potato embryo (StWR/lem), poplar stem (PtWR/1 ca), oat endosperm (AsWR/les), and nutsedge tuber parenchyma (CeWRIItp). (DOCX $15 \mathrm{~kb}$ )

Additional file 2: Amino acid homology of WRI1 homologs.

Comparison of WRI homologs from Arabidopsis (AtWRI1, 3, and 4), poplar stem (PtWRI1 ca), potato embryo (StWRI1em), oat endosperm (AsWRI1es), nutsedge tuber parenchyma (CeWRI1 tp), and tobacco (NbWRI1) in regions spanning the two AP2/EREBP DNA-binding domains. Black means identical, shaded means similarity. Homology is measured as compared to Arabidopsis WRI1 sequence. (JPEG $236 \mathrm{~kb}$ )

Additional file 3: Photo of thin-layer chromatography plate. Lipid classes of total lipid extracts corresponding to $10 \mathrm{mg}$ dw from leaves transiently expressing WRI1 from Arabidopsis embryo (AtWR/1), potato embryo (StWR/1em), oat endosperm (AsWR/les), poplar stem (PtWR/lca), and nutsedge tuber parenchyma (CeWRI1tp) five days after infiltration. Red arrows indicate polar lipids (PL), diacylglycerol (DAG) and triacylglycerol (TAG; oil). Results are from three biological replicates. (TIFF $181 \mathrm{~kb})$

Additional file 4: Dry matter content of leaves. Dry matter as \% dw out of fresh weights of leaves transiently expressing WR/7 from Arabidopsis embryo (AtWR/1), potato embryo (StWR/1em), oat endosperm (AsWR/les), poplar stem (PtWR/1Cm), and nutsedge tuber parenchyma (CeWR/1tp) five days after infiltration. Results are the mean from three biological replicates \pm standard deviation. Letters distinguish significant different means according to Tukey's test at level P $\leq 0.05$. (JPEG $2142 \mathrm{~kb}$ )

Additional file 5: Number of differentially expressed genes (DEGs). Up-regulated and down-regulated genes in leaves expressing WR/7 from Arabidopsis embryo (AtWR/1), potato embryo (StWR/1em), oat endosperm (AsWR/les), poplar stem (PtWR/1ca), and nutsedge tuber parenchyma (CeWR/1tp) in pairwise comparisons to transformed control five days after infiltration. (JPEG $4995 \mathrm{~kb}$ )

Additional file 6: $\log _{2}$ ratios and RPKM values of differentially expressed genes. Table of genes shown to be differential regulated (according to chosen criteria, see Material and Methods) in leaves expressing WRI1 from Arabidopsis embryo (AtWR/1), potato embryo (StWRllem), oat endosperm (AsWRlles), poplar stem $(P t W R / 1 \mathrm{~cm})$, and nutsedge tuber parenchyma $(\mathrm{Ce})$ in pairwise comparisons to transformed control. Genes are grouped into functional categories (CAR; carbohydrates, CYT; cytochromes, DEF; defence, FAS; fatty acid synthesis, GLY; glycolysis, LIP; lipids, MRE; mitochondrial respiration, OTH; other genes, $\mathrm{PHO}$; photosynthesis, PPP; pentose phosphate pathway, STA; starch, STR; stress, TRA; transport, TRF; transcription factors, UNK; unknown. At numbers represent closest Arabidopsis gene homologs of $\mathrm{N}$. benthamiana transcripts from the TAIR database. (XLSX $329 \mathrm{~kb}$ )

Additional file 7: Gene expression pattern of At2g40610 in Arabidopsis. Expression pattern in different tissues according to output image from the developmental series of the Arabidopsis eFP browser with settings in absolute mode. (JPEG $84 \mathrm{~kb}$ )

Additional file 8: Gene expression pattern of At2g22660 (DUF1399) in Arabidopsis. Expression pattern in different tissues according to output image from the developmental series of the Arabidopsis eFP browser with settings in absolute mode. (JPEG 91 kb)

Additional file 9: Time study of starch concentration in leaves expressing AtWRI1. Concentration given as percentage on dry weight (dw) basis from one to five days after infiltration (DAI). TrCtrl, transformed control. Results are the mean from three biological replicates \pm standard deviation. Letters distinguish significant different means according to Tukey's test at level $P \leq 0.05$. (JPEG $2863 \mathrm{~kb}$ )

Additional file 10: Time study of free fatty acid concentration in leaves expressing AtWRI1. Given as nmol fatty acid (FA) per mg dry weight from one to five days after infiltration (DAI). Results are the mean from three biological replicates \pm standard deviation. Letters distinguish significant different means according to Tukey's test at level $\mathrm{P} \leq 0.05$. (JPEG $1958 \mathrm{~kb}$ )

Additional file 11: Primers used in RT-qPCR analyses. (DOCX $14 \mathrm{~kb}$ )

\section{Abbreviations}

ACP: Acyl carrier protein; FA: Fatty acid; KAS: Ketoacyl-ACP synthase; PDCT: Phosphatidylcholine diacylglycerol cholinephosphotransferase; TAG: Triacylglycerol; VLCFA: Very long chain fatty acid; WRI1: WRINKLED1. 


\section{Competing interests}

The authors declare that they have no competing interests.

\section{Authors' contributions}

The design of the study was made by $\mathrm{PH}$, and the experimental setup was planned and performed by $\mathrm{PH}, \mathrm{AC}$, and $\AA \mathrm{G}$. Molecular cloning of genes was performed by $A C, P H, \AA$ G , and RB. Sequence alignments were made by $\mathrm{PH}$. Other experimental procedures, extractions, and analyses were carried out by $\AA$ G,$P H$, and AC. TEM and interpretation of photos was made by SM. PH and $\AA \mathrm{G}$ interpreted the transcriptome data and drafted the manuscript with help from AC. All authors read and approved the final manuscript.

\section{Acknowledgements}

We appreciate the contribution of gene constructs of p19 and GFP from Dr. Craig Wood (CSIRO, Australia). Prof. John Ohlrogge (Michigan, US) is acknowledged for the collaboration in 454 sequencing of nutsedge tubers, and Kerstin Brismar for help with TEM. This work was financially supported by SSF (Swedish Foundation for Strategic Research), VINNOVA (Swedish Governmental Agency for Innovation Systems), and FORMAS (Swedish Research Council for Environment, Agricultural Sciences, and Spatial Planning).

\section{Author details}

${ }^{1}$ Department of Plant Breeding, Swedish University of Agricultural Sciences, Växtskyddsvägen 1, P.O. Box 101, SE-23053 Alnarp, Sweden. ${ }^{2}$ Department of Plant Protection Biology, Swedish University of Agricultural Sciences, Alnarp, Sweden. ${ }^{3}$ Department of Forest Genetics and Plant Physiology, Umeå Plant Science Centre, Umeå, Sweden.

Received: 10 June 2015 Accepted: 23 July 2015

\section{Published online: 08 August 2015}

\section{References}

1. Vanhercke T, Wood CC, Stymne S, Singh SP, Green AG. Metabolic engineering of plant oils and waxes for use as industrial feedstocks. Plant Biotechnol J. 2013;11(2):197-210.

2. Carlsson AS, Yilmaz JL, Green AG, Stymne S, Hofvander P. Replacing fossil oil with fresh oil-with what and for what? Eur J Lipid Sci Technol. 2011;113(7):812-31.

3. Li-Beisson Y, Shorrosh B, Beisson F, Andersson MX, Arondel V, Bates PD, et al. Acyl-lipid metabolism. Arabidopsis Book. 2010;8(10):11.

4. Focks N, Benning C. Wrinkled1: a novel, low-seed-oil mutant of Arabidopsis with a deficiency in the seed-specific regulation of carbohydrate metabolism. Plant Physiol. 1998;118(1):91-101.

5. Cernac A, Benning C. WRINKLED1 encodes an AP2/EREB domain protein involved in the control of storage compound biosynthesis in Arabidopsis. Plant J. 2004;40(4):575-85.

6. Riechmann JL, Meyerowitz EM. The AP2/EREBP family of plant transcription factors. Biol Chem. 1998;379(6):633-46.

7. Ruuska SA, Girke T, Benning C, Ohlrogge JB. Contrapuntal networks of gene expression during Arabidopsis seed filling. Plant Cell. 2002;14(6):1191-206.

8. Maeo K, Tokuda T, Ayame A, Mitsui N, Kawai T, Tsukagoshi H, et al. An AP2-type transcription factor, WRINKLED1, of Arabidopsis thaliana binds to the AW-box sequence conserved among proximal upstream regions of genes involved in fatty acid synthesis. Plant J. 2009;60(3):476-87.

9. Baud S, Wuilleme S, To A, Rochat C, Lepiniec L. Role of WRINKLED1 in the transcriptional regulation of glycolytic and fatty acid biosynthetic genes in Arabidopsis. Plant J. 2009;60(6):933-47.

10. Baud S, Mendoza MS, To A, Harscoet E, Lepiniec L, Dubreucq B. WRINKLED1 specifies the regulatory action of LEAFY COTYLEDON2 towards fatty acid metabolism during seed maturation in Arabidopsis. Plant J. 2007;50(5):825-38.

11. Liu J, Hua W, Zhan G, Wei F, Wang X, Liu G, et al. Increasing seed mass and oil content in transgenic Arabidopsis by the overexpression of wri1-like gene from Brassica napus. Plant Physiol Biochem. 2010;48(1):9-15.

12. Shen B, Allen WB, Zheng P, Li C, Glassman K, Ranch J, et al. Expression of ZmLEC1 and ZmWRI1 increases seed oil production in maize. Plant Physiol. 2010;153(3):980-7.

13. Bourgis F, Kilaru A, Cao X, Ngando-Ebongue GF, Drira N, Ohlrogge JB, et al. Comparative transcriptome and metabolite analysis of oil palm and date palm mesocarp that differ dramatically in carbon partitioning. Proc Natl Acad Sci U S A. 2011;108(30):12527-32.

14. FAOSTAT. ProdSTAT, Crops processed. Rome: Food and Agriculture Organization of the United Nations; 2012.

15. Baud S, Lepiniec L. Physiological and developmental regulation of seed oil production. Prog Lipid Res. 2010;49(3):235-49.

16. Troncoso-Ponce MA, Kilaru A, Cao X, Durrett TP, Fan J, Jensen JK, et al. Comparative deep transcriptional profiling of four developing oilseeds. Plant J. $2011 ; 68(6): 1014-27$.

17. Tranbarger TJ, Dussert S, Joët T, Argout X, Summo M, Champion A, et al Regulatory mechanisms underlying oil palm fruit mesocarp maturation, ripening, and functional specialization in lipid and carotenoid metabolism. Plant Physiol. 2011;156(2):564-84.

18. Turesson H, Marttila S, Gustavsson KE, Hofvander P, Olsson ME, Bulow L, et al. Characterization of oil and starch accumulation in tubers of Cyperus esculentus var. sativus (Cyperaceae): a novel model system to study oil reserves in nonseed tissues. Am J Bot. 2010;97(11):1884-93.

19. Linssen JPH, Cozijnsen JL, Pilnik W. Chufa (cyperus esculentus): a new source of dietary fibre. J Sci Food Agric. 1989;49(3):291-6.

20. Banas A, Debski H, Banas W, Heneen WK, Dahlqvist A, Bafor M, et al. Lipids in grain tissues of oat (Avena sativa): differences in content, time of deposition, and fatty acid composition. J Exp Bot. 2007;58(10):2463-70.

21. Peterson DM, Wood DF. Composition and structure of high-oil oat. J Cereal Sci. 1997;26(1):121-8

22. Hayden DM, Rolletschek H, Borisjuk L, Corwin J, Kliebenstein DJ, Grimberg A, et al. Cofactome analyses reveal enhanced flux of carbon into oil for potential biofuel production. Plant J. 2011;67(6):1018-28.

23. Norlin LH. Tall Oil. In: Ullmann's Encyclopedia of Industrial Chemistry. vol. 35: Wiley-VCH Verlag GmbH \& Co.; 2002: 583-596.

24. Robards AW, Kidwai A. A comparative study of the ultrastructure of resting and active cambium of Salix fragilis L. Planta. 1969;84:239-49.

25. Druart N, Johansson A, Baba K, Schrader J, Sjodin A, Bhalerao RR, et al. Environmental and hormonal regulation of the activity-dormancy cycle in the cambial meristem involves stage-specific modulation of transcriptional and metabolic networks. Plant J. 2007;50(4):557-73.

26. Sjödin A, Street NR, Sandberg G, Gustafsson P, Jansson S. The Populus Genome Integrative Explorer (PopGenIE): a new resource for exploring the Populus genome. New Phytol. 2009;182(4):1013-25.

27. Mu J, Tan H, Zheng Q, Fu F, Liang Y, Zhang J, et al. LEAFY COTYLEDON1 is a key regulator of fatty acid biosynthesis in Arabidopsis. Plant Physiol. 2008;148(2):1042-54

28. To A, Joubes J, Barthole G, Lecureuil A, Scagnelli A, Jasinski S, et al. WRINKLED transcription factors orchestrate tissue-specific regulation of fatty acid biosynthesis in Arabidopsis. Plant Cell. 2012;24(12):5007-23.

29. Ma W, Kong Q, Arondel V, Kilaru A, Bates PD, Thrower NA, et al. WRINKLED1, a ubiquitous regulator in oil accumulating tissues from Arabidopsis embryos to oil palm mesocarp. PLoS ONE. 2013;8(7):e68887.

30. Fan J, Yan C, Zhang X, Xu C. Dual role for phospholipid:diacylglycerol acyltransferase: enhancing fatty acid synthesis and diverting fatty acids from membrane lipids to triacylglycerol in Arabidopsis leaves. Plant Cell. 2013;25(9):3506-18.

31. Vanhercke T, El Tahchy A, Liu Q, Zhou XR, Shrestha P, Divi UK, et al. Metabolic engineering of biomass for high energy density: oilseed-like triacylglycerol yields from plant leaves. Plant Biotechnol J. 2014;12(2):231-9.

32. Hofer R, Dong L, Andre F, Ginglinger JF, Lugan R, Gavira C, et al. Geraniol hydroxylase and hydroxygeraniol oxidase activities of the CYP76 family of cytochrome P450 enzymes and potential for engineering the early steps of the (seco)iridoid pathway. Metab Eng. 2013;20:221-32.

33. Liu Q, Manzano D, Tanic N, Pesic M, Bankovic J, Pateraki l, et al. Elucidation and in planta reconstitution of the parthenolide biosynthetic pathway. Metab Eng. 2014;23:145-53.

34. Petrie JR, Shrestha P, Mansour MP, Nichols PD, Liu Q, Singh SP. Metabolic engineering of omega-3 long-chain polyunsaturated fatty acids in plants using an acyl-CoA Delta6-desaturase with omega3-preference from the marine microalga Micromonas pusilla. Metab Eng. 2010;12(3):233-40.

35. Wood CC, Petrie JR, Shrestha P, Mansour MP, Nichols PD, Green AG, et al. A leaf-based assay using interchangeable design principles to rapidly assemble multistep recombinant pathways. Plant Biotechnol J. 2009;7(9):914-24.

36. Lonien J, Schwender J. Analysis of metabolic flux phenotypes for two arabidopsis mutants with severe impairment in seed storage lipid synthesis. Plant Physiol. 2009;151(3):1617-34. 
37. Baud S, Graham IA. A spatiotemporal analysis of enzymatic activities associated with carbon metabolism in wild-type and mutant embryos of Arabidopsis using in situ histochemistry. Plant J. 2006;46(1):155-69.

38. Lu C, Xin Z, Ren Z, Miquel M, Browse J. An enzyme regulating triacylglycerol composition is encoded by the ROD1 gene of Arabidopsis. Proc Natl Acad Sci. 2009;106(44):18837-42.

39. Nandi A, Welti R, Shah J. The Arabidopsis thaliana dihydroxyacetone phosphate reductase gene SUPPRESSSOR OF FATTY ACID DESATURASE DEFICIENCY1 is required for glycerolipid metabolism and for the activation of systemic acquired resistance. Plant Cell. 2004;16(2):465-77.

40. Wei Y, Periappuram C, Datla R, Selvaraj G, Zou J. Molecular and biochemical characterizations of a plastidic glycerol-3-phosphate dehydrogenase from Arabidopsis. Plant Physiol Biochem. 2001;39(10):841-8.

41. Kawai H, Ishikawa T, Mitsui T, Kore-eda S, Yamada-Kawai M, Ohnishi J-i. Arabidopsis glycerol-3-phosphate permease 4 is localized in the plastids and involved in the accumulation of seed oil. Plant Biotechnology. 2014;31(2):15965.

42. Pouvreau B, Baud S, Vernoud V, Morin V, Py C, Gendrot G, et al. Duplicate maize Wrinkled 1 transcription factors activate target genes involved in seed oil biosynthesis. Plant Physiol. 2011;156(2):674-86.

43. Winter D, Vinegar B, Nahal H, Ammar R, Wilson GV, Provart NJ. An "Electronic Fluorescent Pictograph" browser for exploring and analyzing large-scale biological data sets. PLoS ONE. 2007;2(8):e718.

44. Baud S, Dubreucq B, Miquel M, Rochat C, Lepiniec L. Storage reserve accumulation in arabidopsis: metabolic and developmental control of seed filling. The Arabidopsis Book. 2008;6:e0113.

45. Weber AP, Linka N. Connecting the plastid: transporters of the plastid envelope and their role in linking plastidial with cytosolic metabolism. Annu Rev Plant Biol. 2011;62:53-77.

46. Flugge UI, Hausler RE, Ludewig F, Gierth M. The role of transporters in supplying energy to plant plastids. J Exp Bot. 2011;62(7):2381-92.

47. Da Silva PMFR, Eastmond PJ, Hill LM, Smith AM, Rawsthorne S. Starch metabolism in developing embryos of oilseed rape. Planta. 1997;203(4):480-7.

48. Lu S, Song T, Kosma DK, Parsons EP, Rowland O, Jenks MA. Arabidopsis CER8 encodes LONG-CHAIN ACYL-COA SYNTHETASE 1 (LACS1) that has overlapping functions with LACS2 in plant wax and cutin synthesis. Plant J. 2009;59(4):553-64.

49. Fiebig A, Mayfield JA, Miley NL, Chau S, Fischer RL, Preuss D. Alterations in CER6, a gene identical to CUT1, differentially affect long-chain lipid content on the surface of pollen and stems. Plant Cell. 2000;12(10):2001-8.

50. Pascal S, Bernard A, Sorel M, Pervent M, Vile D, Haslam RP, et al. The Arabidopsis cer26 mutant, like the cer2 mutant, is specifically affected in the very long chain fatty acid elongation process. Plant J. 2013;73(5):733-46.

51. Aarts MG, Keijzer CJ, Stiekema WJ, Pereira A. Molecular characterization of the CER1 gene of arabidopsis involved in epicuticular wax biosynthesis and pollen fertility. Plant Cell. 1995;7(12):2115-27.

52. Panikashvili D, Shi JX, Schreiber L, Aharoni A. The Arabidopsis DCR encoding a soluble BAHD acyltransferase is required for cutin polyester formation and seed hydration properties. Plant Physiol. 2009;151(4):1773-89.

53. Li Y, Beisson F, Koo AJK, Molina I, Pollard M, Ohlrogge J. Identification of acyltransferases required for cutin biosynthesis and production of cutin with suberin-like monomers. Proc Natl Acad Sci. 2007;104(46):18339-44.

54. Xiao F, Goodwin SM, Xiao Y, Sun Z, Baker D, Tang X, et al. Arabidopsis CYP86A2 represses Pseudomonas syringae type III genes and is required for cuticle development. EMBO J. 2004:23(14):2903-13.

55. Eastmond PJ. Glycerol-insensitive Arabidopsis mutants: gli1 seedlings lack glycerol kinase, accumulate glycerol and are more resistant to abiotic stress. Plant J. 2004;37(4):617-25.

56. Eastmond PJ, Dennis DT, Rawsthorne S. Evidence that a malate/inorganic phosphate exchange translocator imports carbon across the leucoplast envelope for fatty acid synthesis in developing castor seed endosperm. Plant Physiol. 1997;114(3):851-6.

57. Neuhaus HE, Emes MJ. Nonphotosynthetic metabolism in plastids. Annu Rev Plant Physiol Plant Mol Biol. 2000:51:111-40.

58. Schaffer JE. Lipotoxicity: when tissues overeat. Curr Opin Lipidol. 2003;14(3):281-7.

59. Eastmond PJ, van Dijken AJ, Spielman M, Kerr A, Tissier AF, Dickinson HG, et al. Trehalose-6-phosphate synthase 1, which catalyses the first step in trehalose synthesis, is essential for Arabidopsis embryo maturation. Plant J. 2002;29(2):225-35.
60. Gomez LD, Baud S, Gilday A, Li Y, Graham IA. Delayed embryo development in the ARABIDOPSIS TREHALOSE-6-PHOSPHATE SYNTHASE 1 mutant is associated with altered cell wall structure, decreased cell division and starch accumulation. Plant J. 2006:46(1):69-84.

61. Gomez LD, Baud S, Graham IA. The role of trehalose-6-phosphate synthase in Arabidopsis embryo development. Biochem Soc Trans. 2005;33(Pt 1):280-2.

62. Bao F, Azhakanandam S, Franks RG. SEUSS and SEUSS-LIKE transcriptional adaptors regulate floral and embryonic development in Arabidopsis. Plant Physiol. 2010;152(2):821-36.

63. Jolivet P, Roux E, d'Andrea S, Davanture M, Negroni L, Zivy M, et al. Protein composition of oil bodies in Arabidopsis thaliana ecotype WS. Plant Physiol Biochem. 2004;42(6):501-9.

64. Liu D, Shi L, Han C, Yu J, Li D, Zhang Y. Validation of reference genes for gene expression studies in virus-infected Nicotiana benthamiana using quantitative real-time PCR. PLOS ONE. 2012;7(9):e46451.

65. Santos-Mendoza M, Dubreuca B, Baud S, Parcy F, Caboche M, Lepiniec L. Deciphering gene regulatory networks that control seed development and maturation in Arabidopsis. Plant J. 2008;54(4):608-20.

66. Bligh EG, Dyer WJ. A rapid method of total lipid extraction and purification. Can J Biochem Physiol. 1959:37(8):911-7.

67. Leonova S, Shelenga T, Hamberg M, Konarev AV, Loskutov I, Carlsson AS. Analysis of oil composition in cultivars and wild species of oat (Avena sp.). J Agric Food Chem. 2008;56(17):7983-91.

68. Leonova S, Grimberg Å, Marttila S, Stymne S, Carlsson AS. Mobilization of lipid reserves during germination of oat (Avena sativa L.), a cereal rich in endosperm oil. J Exp Bot. 2010;61(11):3089-99.

69. Lamesch P, Berardini TZ, Li D, Swarbreck D, Wilks C, Sasidharan R, et al. The Arabidopsis Information Resource (TAIR): improved gene annotation and new tools. Nucleic Acids Res. 2012;40(D1):D1202-10.

\section{Submit your next manuscript to BioMed Central and take full advantage of:}

- Convenient online submission

- Thorough peer review

- No space constraints or color figure charges

- Immediate publication on acceptance

- Inclusion in PubMed, CAS, Scopus and Google Scholar

- Research which is freely available for redistribution 